SUPPLEMENT ARTICLE

\title{
The Safety Profile of Varicella Vaccine: A 10-Year Review
}

\author{
Susan A. Galea, ${ }^{1}$ Ann Sweet, ${ }^{1}$ Paul Beninger, ${ }^{1}$ Sharon P. Steinberg, ${ }^{2}$ Philip S. LaRussa, ${ }^{2}$ Anne A. Gershon, ${ }^{2}$ \\ and Robert G. Sharrar ${ }^{1}$ \\ ${ }^{1}$ Merck Research Laboratories, Clinical Risk Management and Safety Surveillance, North Wales, Pennsylvania; ${ }^{2}$ Columbia University \\ College of Physicians and Surgeons, New York, New York
}

Varivax (varicella virus vaccine live [Oka/Merck]; Merck), a live attenuated varicella vaccine, is indicated for vaccination against varicella in appropriate individuals $\geqslant 12$ months of age. The 10 -year safety profile for Varivax is described using data submitted to Merck from routine global postmarketing surveillance, combined with information from a Varicella Zoster Virus Identification Program, which uses polymerase chain reaction (PCR) analysis to identify the presence and strain of VZV in selected specimens. There were 16,683 reports worldwide voluntarily submitted to Merck, for an overall reporting rate of 3.4 reports/10,000 doses of vaccine distributed. PCR analysis of vesicular rashes that occurred within the first 2 weeks after vaccination was more likely to identify wild-type varicella-zoster virus (VZV), whereas the presence of Oka VZV was generally associated with vesicular rashes that occurred 15-42 days after vaccination. Reports of breakthrough varicella that occurred $>42$ days after vaccination were associated with wild-type VZV. Among 697 herpes zoster reports, PCR analysis identified Oka VZV in 57 reports and wild-type VZV in 38 reports. There were no primary neurologic adverse events associated with Oka VZV. Secondary transmission of Oka VZV from vaccine recipients with postvaccination vesicular rashes was identified in 3 susceptible household contacts. Disseminated Oka VZV was identified in 6 immunocompromised patients and 1 patient with Down syndrome. This review has shown that the vaccine is generally safe and well tolerated.

Varivax (varicella virus vaccine live [Oka/Merck]; Merck), a live attenuated viral vaccine, became commercially available in the United States on 1 May 1995. The vaccine has been marketed worldwide in 46 countries, with $\sim 55$ million doses distributed.

In the present article, we describe the safety profile of varicella vaccine by use of spontaneous postmarketing adverse experience (AE) reports submitted to

Potential conflicts of interest: S.A.G., A.S., P.B., and R.G.S. are salaried employees of Merck and possess stock and stock options in the company. A.A.G. lectures and consults on varicella-zoster virus vaccines for Merck and GlaxoSmithKline when invited and receives research support from Merck. Additionally, P.S.L., S.P.S., and A.A.G. are in a contractual relationship with Merck through the Varicella Zoster Virus Identification Program.

Financial support: supplement sponsorship is detailed in the Acknowledgments. Reprints or correspondence: Susan A. Galea, Merck Research Laboratories, Clinical Risk Management and Safety Surveillance, UG3C-54, PO Box 1000, North Wales, PA 19454-1099 (susan_galea@merck.com).

The Journal of Infectious Diseases 2008; 197:S165-9

(C) 2008 by the Infectious Diseases Society of America. All rights reserved. 0022-1899/2008/19705S2-0025\$15.00

DOI: $10.1086 / 522125$
Merck, together with results from the Varicella Zoster Virus Identification Program (VZVIP) during the first 10 years of marketing experience since the licensure of Varivax.

\section{METHODS}

Postmarketing surveillance. The postmarketing reporting system for AEs is a passive, spontaneous, voluntary, incomplete reporting system. Merck's Worldwide Adverse Experience System database contains records of AEs spontaneously reported to the company by health care professionals and consumers, as well as case reports from the published literature. All reported AEs temporally related to the administration of a vaccine are added to the database without regard to the likelihood of a causal relationship and are coded in the terminology used by the reporter.

Definitions. The following case definitions were used to categorize AE data for analysis. A breakthrough 
Table 1. Varicella Zoster Virus Identification Program polymerase chain reaction results.

\begin{tabular}{lccccrr}
\hline AE of interest & Oka VZV & $\begin{array}{c}\text { Wild-type } \\
\text { VZV }\end{array}$ & $\begin{array}{c}\text { VZV } \\
\text { negative }\end{array}$ & $\begin{array}{c}\text { VZV positive } \\
\text { and }\end{array}$ & $\begin{array}{c}\text { Inadequate } \\
\text { sample }\end{array}$ & Total \\
\hline Rash $\leqslant 42$ days after vaccination & 37 & 42 & 17 & 9 & 25 & 130 \\
Breakthrough varicella $^{\text {Herpes zoster }}$ & 0 & 16 & 10 & 1 & 6 & 33 \\
Neurologic complications $^{\text {a }}$ & 57 & 38 & 16 & 4 & 47 & 162 \\
Secondary transmission $_{\quad}$ & 0 & 1 & 27 & 1 & 1 & 30 \\
$\quad$ Total & 3 & 30 & 9 & 0 & 6 & 48 \\
\hline
\end{tabular}

NOTE. Data are no. of specimens. VZV, varicella-zoster virus.

a The herpes zoster specimens include 6 reported cases of herpes zoster with mild meningeal symptoms/meningitis, which are included here under herpes zoster and are discussed in detail in the "HZ" subsection of Results.

case of varicella was defined as a varicella-like rash (chickenpox) in a vaccine recipient that occurred $>42$ days after vaccination [1]. A secondary transmission case was defined as the documented presence of Oka VZV in a nonvaccinated contact of an individual vaccinated with Varivax.

VZVIP. VZVIP uses polymerase chain reaction (PCR) analysis to distinguish the Oka VZV strain from the wild-type VZV strain circulating in the United States. Clinical specimens were collected from patients with $>50$ vesicular lesions occurring within 42 days of vaccination, breakthrough cases of varicella, herpes zoster (HZ), encephalitis, aseptic meningitis, cerebellar ataxia, pneumonitis, and suspected secondary transmission. PCR analysis and sample collection have been described elsewhere $[1,2]$.

\section{RESULTS}

From 1 May 1995 through 30 April 2005, 55.7 million doses of Varivax were distributed worldwide. Merck received 16,683 $\mathrm{AE}$ reports after the administration of varicella vaccine, for an overall reporting rate of $3.4 \mathrm{AEs} / 10,000$ doses distributed (database query, 8 August 2005). AEs of interest that will be reviewed here include rash within 42 days of vaccination, breakthrough varicella, HZ, neurologic AEs, and secondary transmission of Oka VZV. The PCR results for the AEs of interest are presented in table 1.

Rashes within 42 days of vaccination. There were 3192 reports describing rashes occurring within 42 days after vaccination. Fifty-one percent were varicella-like rashes, $41 \%$ were not well described or were nonspecific rashes, and $\sim 8 \%$ were injection site rashes/vesicles. Sixty-five percent of the reported rashes occurred within the first 14 days of receiving the vaccine, and $89 \%$ occurred within the first 21 days.

The wild-type VZV rashes occurred a median of 8 days (range, 1-20 days) after vaccination, whereas the Oka VZV rashes occurred at a median of 21 days (range, 5-42 days) after vaccination. The median number of lesions with wild-type VZV rashes was 100 (range, 10-1000), whereas the median number of lesions with Oka VZV rashes was 51 (range, 1-500). Thirty- six $(84 \%)$ of the $43 \mathrm{VZV}$ rashes that occurred within the first 2 weeks after vaccination were identified as wild-type VZV, and $30(83 \%)$ of 36 VZV rashes that occurred within 3-6 weeks were identified as the Oka VZV strain (figure 1).

Eight of the patients in whom Oka VZV was identified in a rash occurring $<42$ days after vaccination were immunocompromised. These included patients with undiagnosed HIV infection, patients with undiagnosed primary immunodeficiency, transplant recipients receiving high-dose steroids, and patients with newly diagnosed systemic lupus erythematosus $[1,3,4]$. Additionally, of the 5 specimens with identified Oka VZV in the fifth and sixth week after vaccination, 3 were from patients who were immunocompromised, and 1 was from a patient who had a history of Marfan syndrome.

Reports of breakthrough varicella. There were 5054 reports of breakthrough varicella, for a reporting rate of 0.9 reports/10,000 doses of vaccine distributed. Fifty-one reports (1\%) met the regulatory definition of "serious." Thirty-eight reports occurred in immunocompetent hosts, and 13 occurred in immunocompromised patients. Of the 38 immunocompetent hosts, 17 developed varicella described as "severe," "fullblown," or "rash" (2 included vomiting and dehydration); 17 required treatment for secondary infection (cellulitis [12 patients], pneumonia [ 2 patients], sepsis [ 2 patients], and keratitis

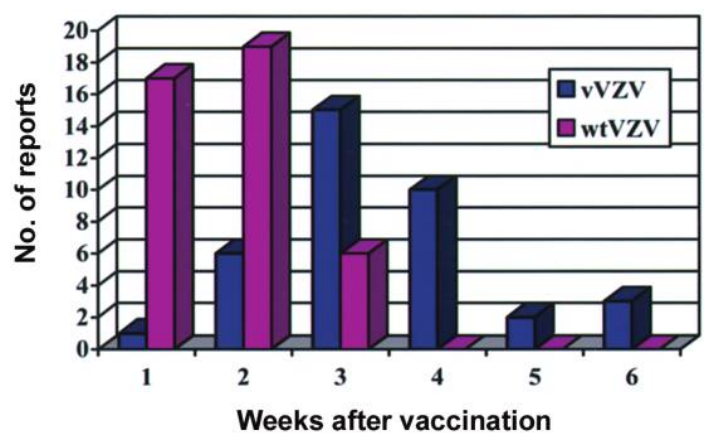

Figure 1. Time to onset of rashes identified as $0 k$ a and wild-type varicella-zoster virus (vVZV and wtVZV, respectively). 
[1 patient]); 3 had neurologic complications (ataxia [2 patients] and aseptic meningitis [ 1 patient]), and 1 experienced myocarditis with varicella. Oka VZV was not identified in any specimens from breakthrough varicella.

The 13 reports in immunocompromised hosts occurred 50 days to 7 years after vaccination. The immunocompromising conditions included cancer/leukemia (5 patients), use of highdose steroids to treat underlying conditions (4 patients), transplant recipients ( 2 patients), undiagnosed primary immunodeficiency ( 1 patients), and failure to thrive ( 1 patient). Of the 7 specimens ( 4 of the 7 were analyzed by outside laboratories and not through the VZVIP), 5 were positive for wild-type VZV, and 2 were VZV positive but could not be further typed/ identified. Nine patients recovered, and the outcome in 1 case is not reported. Three patients died of disseminated VZV infection 1.7-7 years after vaccination (2 of the 3 specimens were positive for wild-type VZV, and 1 was VZV positive, strain not identified).

HZ. There were 697 reports of HZ occurring 1-3509 days (median, 362 days) after vaccination in patients 13 months to 68 years of age (median age, 3.4 years). Four hundred patients $(65 \%)$ for whom age was reported were $<5$ years of age. Table 2 compares the HZ reports in which PCR analysis identified Oka VZV and wild-type VZV strains.

The site of $\mathrm{HZ}$ was more likely to correlate with the site of vaccine injection in reports in which Oka VZV was identified. PCR was more likely to identify wild-type VZV than Oka VZV if the time to onset was within 42 days of vaccination. However, 2 reports in which $\mathrm{HZ}$ was diagnosed within 42 days of vaccination had specimens in which Oka VZV was identified. One of these reports was of a child with acute lymphocytic leukemia diagnosed 10 days after vaccination with Varivax. The child eventually developed HZ on 3 occasions: 23, 47, and 116 days after vaccination. PCR analysis of a specimen from the last episode of HZ identified Oka VZV. In the second report, Oka VZV was present in a girl 5 years of age who developed an HZ-like rash in the distribution of the second division of the trigeminal nerve (right side of face and right eye) 25 days after receiving Varivax.
Five patients developed meningitis in association with $\mathrm{HZ}$. Cerebrospinal fluid (CSF) specimens from these patients were negative for VZV. The HZ rash specimens from 2 of the patients had Oka VZV identified; however, in 1 of these reports, enterovirus was identified in the CSF analyzed at the Centers for Disease Control and Prevention (CDC). A third patient had wild-type VZV identified from an $\mathrm{HZ}$ rash specimen.

Additionally, 1 child who received Varivax at 2-3 years of age had acute lymphocytic leukemia diagnosed at 4 years of age. After treatment with 6-mercaptopurine weekly and methotrexate monthly, he was hospitalized for $\mathrm{HZ}$ and mild meningeal signs. A CSF specimen had Oka VZV identified. The child recovered.

Neurologic AEs. Neurologic syndromes, such as encephalitis, aseptic meningitis, and cerebellar ataxia, have been reported in the postmarketing environment after administration of Varivax. There were 30 (CSF) specimens analyzed by PCR (table 1) from patients with reports of such syndromes. The reported AEs associated with these reports included encephalitis (12 patients), meningitis ( 5 patients), ataxia ( 5 patients), transverse myelitis ( 3 patients), seizures (3 patients), demyelinating disorder ( 1 patient), and hemiparesis ( 1 patient). The 5 meningitis reports associated with $\mathrm{HZ}$ listed in the "HZ" subsection above are different from the cases of meningitis listed in this subsection and are not included here. None of the CSF specimens from these neurologic reports had Oka VZV identified by PCR analysis. Several of these reports have been described elsewhere $[1,4]$.

Secondary transmission. The VZVIP confirmed 3 cases of secondary transmission of Oka VZV. The Oka VZV was present in a 30-year-old pregnant woman who developed 100 vesicular lesions 16 days after her 1-year-old son developed $\sim 30$ vesicular lesions 24 days after varicella vaccination. She elected to have a therapeutic abortion, and the products of conception were negative for VZV by PCR analysis [5]. In the second report, the Oka VZV strain was also present in a 4-month-old boy who developed 25 lesions 19 days after his 1-year-old sibling developed 2 vesicular lesions 14 days after vaccination. The third case in which Oka VZV was identified occurred in a 35-

Table 2. Comparison of Oka varicella-zoster virus (VZV) and wild-type VZV herpes zoster (HZ) specimens.

\begin{tabular}{lcc}
\hline Variable & $\begin{array}{c}\text { Oka } \\
\text { VZV }\end{array}$ & $\begin{array}{c}\text { Wild-type } \\
\text { VZV }\end{array}$ \\
\hline Patients, no. & 57 & 38 \\
Age of patients, median (range), years & $2(1-9)$ & $4(1-55)$ \\
Time to onset of reported $\mathrm{HZ}$ from vaccination, median (range) & 318 days (23 days- & 588 days (4 days- \\
& 7.7 years) & 7.8 years) \\
Patients with postvaccination rash $\leqslant 42$ days after vaccination, no. (\%) & $3(5.3)$ & 0 \\
Site of injection correlated with the site of $\mathrm{HZ}$, no. (\%) & $26(46)$ & $5(13.2)$ \\
Patients with $\mathrm{HZ}$ occurring $\leqslant 42$ days after vaccination, no. & 2 & 10 \\
Patients with $\mathrm{HZ}$ occurring $>42$ days after vaccination, no. & 55 & 28 \\
\hline
\end{tabular}


year-old father who developed $>100$ lesions 17 days after his 1 -year-old son developed 12 vesicular lesions 17 days after vaccination. In each of the 3 confirmed secondary-transmission cases, the vaccine recipient had a postvaccination rash and had close, household contact with the susceptible individual.

Additionally, there were 2 reported cases of possible secondary transmission, in which it was reported that the presence of Oka VZV was identified by an outside laboratory [6, 7]. The specimens for these cases were not analyzed through or confirmed by the VZVIP.

Disseminated Oka VZV infection. There were 7 reports of disseminated VZV in which Oka VZV was identified. These occurred in 6 patients with immunodeficiency disorders and 1 patient with Down syndrome. The 7 reports are described briefly below.

1. A 13-month-old boy with a history of oral thrush for 3 months before vaccination developed a vesicular, dermatomal rash 15 days after receiving Varivax, followed by respiratory distress 83 days after vaccination. He was subsequently found to be HIV positive [5]. PCR analysis of a bronchoalveolar lavage (BAL) sample revealed the presence of Oka VZV.

2. A 5-year-old boy with severe cerebral palsy, seizure disorder, quadriplegia, and reactive airway disease treated with intermittent steroid therapy was vaccinated 7 days after finishing a steroid taper. Eight days later, the child restarted steroid therapy; 2 days later, he developed a rash that was followed 7 days later by pneumonia. PCR analysis identified Oka VZV in endotracheal secretions [1].

3. A 13-month-old boy with failure to thrive, recurrent bronchiolitis, and oral candidiasis was hospitalized 10 days after vaccination in respiratory distress with an enlarged liver and elevated hepatic enzymes. A liver biopsy showed multifocal necrosis, and Oka VZV was identified. He subsequently developed varicella and later received a diagnosis of combined immune deficiency caused by an adenosine deaminase deficiency [1].

4. An 11-year-old girl with mental retardation; a history of congenital cytomegalovirus infection; and recurrent, presumably viral, respiratory infections presented with a diffuse vesicular rash and respiratory distress $\sim 5$ weeks after vaccination. She received a diagnosis of "post vaccine varicella pneumonitis" and was subsequently diagnosed with a novel immunodeficiency of NK T cells $[8,9]$. PCR analyses of BAL and endotracheal secretion specimens identified the presence of Oka VZV.

5. A 13-month-old boy with DiGeorge syndrome postcentral shunt and Rastelli repair for tetralogy of Fallot was vaccinated with Varivax and measles-mumps-rubella vaccine (M-M-R II; Merck); 27 days later, he developed diffuse bilateral pneumonitis. The patient was hospitalized 7 days later with severe anemia, congestive heart failure, and bloody emesis. He subsequently developed a vesicular rash with 5 lesions scattered on the trunk. PCR analysis of vesicular and bronchial washings identified Oka VZV. The CDC also reported that the tracheal aspirate was positive for measles virus. The patient subsequently died. Results of PCR testing of autopsy samples from the lungs and lymph nodes were negative for VZV.

6. A 14-month-old boy with asthma developed a vesicular rash 19 days after vaccination and was subsequently hospitalized with a disseminated rash, fevers, and elevated aspartate aminotransferase and alanine aminotransferase levels. He had treatment initiated with intravenous acyclovir. A liver biopsy revealed multinucleated giant cells consistent with VZV infection, and an abdominal sonogram was consistent with visceral dissemination. The child was diagnosed with a severe combined immunodeficiency. PCR analysis of a lesion scab revealed the presence of Oka VZV.

7. A 48-year-old male patient with Down syndrome developed a fever of $41.2^{\circ} \mathrm{C}$ and was hospitalized with pneumonitis 13 days after vaccination. Two weeks after admission, he developed a generalized rash. PCR results from lesion and sputum specimens were positive for the Oka VZV strain. However, the sputum was not from BAL and may have been contaminated by oral lesions.

\section{DISCUSSION}

Although it has limitations, the strengths of postmarketing surveillance include the following: it collects information on a large number of individuals across a wide range of medical practices, it provides data regarding unstudied uses or populations, and it can detect rare, unexpected AEs [10]. Ten years of postmarketing surveillance of Varivax has been enhanced by the data obtained through the VZVIP. The AEs described include rashes within 42 days after vaccination, breakthrough reports of varicella, HZ, neurologic AEs, secondary transmission, and disseminated Oka VZV cases.

With respect to vesicular rashes within 42 days after vaccination, PCR analysis is more likely to identify wild-type VZV in those rashes occurring within the first 2 weeks after vaccination, whereas rashes occurring 15-42 days after vaccination are more likely to be identified as Oka VZV. This trend is similar to what has been previously reported [1].

Reports of varicella that occurred $>42$ days after vaccination were associated with wild-type VZV. The majority (99\%) of these breakthrough reports were not serious by regulatory definition.

Regarding reported HZ cases, when comparing Oka VZV cases with wild-type VZV cases, those identified as Oka VZV tended to occur more often in younger children (median age, 2 vs. 4 years), with a shorter median time to onset after vaccination (318 vs. 588 days) and with the site of $\mathrm{HZ}$ correlating more often with the site of vaccination ( $46 \%$ vs. $13.2 \%$ ). 
Reported neurological AEs after varicella vaccination included encephalitis, meningitis, and cerebellar ataxia. There have not been any primary neurologic cases in which PCR analysis has identified the presence of Oka VZV in a normal host.

The VZVIP has documented 3 cases of secondary transmission of the Oka VZV from a vaccine recipient to an unvaccinated susceptible host. In all 3 instances, the vaccine recipient experienced a rash and had close, household contact with the susceptible individual.

There were 7 reports of disseminated Oka VZV reported in 6 immunocompromised patients and 1 patient with Down syndrome. Vaccination of individuals receiving immunosuppressive therapy or with primary or acquired immunodeficiencies is contraindicated, per the package circular for Varivax.

This 10-year review of spontaneous reporting after distribution of $>55$ million doses of Varivax demonstrates that the interpretation of postmarketing $\mathrm{AE}$ reports has been enhanced by the VZVIP and that Varivax is generally well tolerated.

\section{Acknowledgments}

Supplement sponsorship. This article was published as part of a supplement entitled "Varicella Vaccine in the United States: A Decade of Prevention and the Way Forward," sponsored by the Research Foundation for Microbial Diseases of Osaka University, GlaxoSmithKline Biologicals, the
Sabin Vaccine Institute, the Centers for Disease Control and Prevention, and the March of Dimes.

\section{References}

1. Sharrar RG, LaRussa P, Galea SA, et al. The postmarketing safety profile of varicella vaccine. Vaccine 2001; 19:916-23.

2. LaRussa P, Lungu O, Hardy I, Gershon A, Steinberg S, Silverstein S. Restriction fragment length polymorphism of polymerase chain reaction products from vaccine and wild-type varicella-zoster virus isolates. J Virol 1992; 66:1016-20.

3. Ardron P, LaRussa P, Steinberg S, Caserta M. Severe vaccine-associated rash following varicella vaccine (VARIVAX ${ }^{(\mathbb{M})}$ ) in an adolescent with primary sclerosing cholangitis. Pediatr Res 1996;39(4 Part 2):165A.

4. Wise R, Salive, M, Braun M, et al. Postlicensure safety surveillance for varicella vaccine. JAMA 2000;284:1271-9.

5. Salzman MB, Sharrar RG, Steinberg S, LaRussa P. Transmission of varicella-vaccine from a healthy 12 -month-old to his pregnant mother. J Pediatr 1997; 131:151-4.

6. Brunell $\mathrm{P}$, Takele A. Chickenpox attributable to a vaccine virus contracted from a vaccinee with zoster. Pediatrics 2000; 106:1-2.

7. Grossman R, Harpaz R, Rubtcova E, Loparev V, Seward JF, Schmid DS. Secondary transmission of varicella vaccine virus in a chronic care facility for children. J Pediatr 2006; 148:842-4.

8. Levy O, Orange JS, Hibberd P, et al. Disseminated varicella infection due to vaccine strain of varicella-zoster virus in a patient with a novel deficiency in natural killer T cells. J Infect Dis 2003; 188:948-53.

9. Gershon A. Varicella vaccine: rare serious problems-but the benefits still outweigh the risks. J Infect Dis 2003; 188:945-7.

10. Kennedy D, Goldman S, Lillie R. Spontaneous reporting in the United States. In: Strom BL, ed. Pharmacoepidemiology. 3rd ed. Chicester, United Kingdom: Wiley, 2000:151-74. 